\title{
$\begin{array}{ll}\text { Research Square } & \text { Preprints are preliminary reports that have not undergone peer review. } \\ \text { They should not be considered conclusive, used to inform clinical practice, }\end{array}$ or referenced by the media as validated information. \\ The influence of Discoidin Domain Receptor 1 expression on angiogenic factors: VEGF-A and FGF- 2 in non-small cell lung cancer
}

\section{Zahra Damavandi}

National Institute for Genetic Engineering and Biotechnology

\section{Pardis Riahi}

National Institute for Genetic Engineering and Biotechnology

Tayebeh Majidizadeh

National Institute for Genetic Engineering and Biotechnology

massoud houshmand ( $\nabla$ massoudh@nigeb.ac.ir)

National Institute for Genetic Engineering and Biotechnology https://orcid.org/0000-0002-2198-223X

\section{Research Article}

Keywords: DDR1, collagen type I, non-small cell lung cancer, angiogenesis, VEGF, FGF

Posted Date: February 23rd, 2022

DOI: https://doi.org/10.21203/rs.3.rs-1334523/v1

License: (c) (i) This work is licensed under a Creative Commons Attribution 4.0 International License.

Read Full License 


\section{Abstract}

Purpose: Lung cancer is responsible for more cancer-related death worldwide Most lung cancer patients have non-small cell lung cancers (NSCLC), with low survival rate. Therefore, it is necessary to study new and effective targeted therapies. Discoidin domain receptor1 (DDR1) is a tyrosine kinase receptor that reacts to various collagens. Expression of DDR1 can be promoted by collagen type l, and dysregulation of DDR1 signaling are involved in several phases of tumorigenesis. Tumor growth and metastasis depend on angiogenesis caused by angiogenic factors, such as vascular endothelial growth factor (VEGF) and fibroblast growth factor (FGF) secreted by tumor cells. Thus, Angiogenesis plays a critical role in cancer progression and inhibition of angiogenesis is essential for cancer treatment. The aim of the current study was to evaluate the alteration of DDR1 expression and its effects on VEGF-A, FGF-1, and FGF2 in NSCLC.

Methods: A549 and Calu-3 cell lines, were treated with collagen type I and transfected with DDR1 small interfering RNA (siRNA). Then, the relative expression of DDR1, VEGF-A, FGF-1, and FGF-2 was evaluated using quantitative real-time polymerase chain reaction (qRT-PCR). The concentration of angiogenic factors was calculated by Human Angiogenesis 17-plex discovery assay.

Results: According to our data, the collagen type I could stimulate DDR1 expression and DDR1 upregulation resulted in a significant increase of VEGF-A and FGF-2 expression, but did not induce FGF-1 expression. Moreover, DDR1 downregulation significantly decreased VEGF-A and FGF-2 expression.

Conclusion: our findings suggest that alteration of DDR1 expression may affect VEGF-A and FGF-2 expression in NSCLC associated with tumor angiogenesis and represent a new treatment approach.

\section{Introduction}

Lung cancer is the leading cause of cancer-related death worldwide. Lung cancer comprises two main histologic subtypes: non-small cell lung cancer (NSCLC) and small cell lung cancer (SCLC). NSCLC, which includes large-cell carcinoma, squamous cell carcinoma, and adenocarcinoma, is the predominant form of lung cancer [1, 2]. Despite many advances in several fields of oncology, lung cancer treatment has not progressed significantly. Understanding the biological pathways involved in the cause of lung cancer is needed to recognize important biomolecules as diagnostic markers and new targeted therapies [3].

Discoidin domain receptors (DDRs) are kinds of tyrosine kinase receptors (RTKs) that have an extracellular discoidin homology domain for collagen binding and activation. DDR1 and DDR2 are two types of DDRs. The only RTKs that recognize specific amino acid motifs in collagen (binding motif: GVMGFO, six amino acid) and collagen activate them are the DDRs. The DDRs are important transmembrane regulators that, as part of extracellular matrix networks, regulate signaling and cell-matrix interactions. Therefore, the DDRs are involved in cancer progression by controlling the interactions between tumor cells and the collagen matrix around them [4-7]. 
Discoidin domain receptor 1 gene has 17 exons that are alternatively spliced to generate five DDR1 isoforms $(a-e)$ that differ in the cytoplasmic region. DDR1 is widely distributed in the body but is expressed mainly in epithelial cells and is activated by fibrillar and non-fibrillar collagens. DDR1 signaling has been indicated to play a critical role in various cellular functions such as ECM remodeling, proliferation, survival, migration, immune response, differentiation, and wound healing. Different studies have shown DDR1 expression in a variety of human cancers such as renal clear cell carcinoma, breast cancer, non-small cell lung carcinoma, prostate cancer, esophageal cancer, and hepatocellular carcinoma indicating the function of DDR1 in tumor progression [7-12].

Angiogenesis is a vital process required for a variety of physiological and pathological functions, such as inflammation, metabolic diseases, wound repair, and tumor progression. As cancer progresses, angiogenesis is important to provide nutrition and remove waste products for tumor proliferation, survival and metastasis. Many different proteins have been discovered as proangiogenic and antiangiogenic molecules that regulate angiogenesis and their expression level determines the tumor cells invasion. Proangiogenic factors, such as vascular endothelial growth factor (VEGF) and fibroblast growth factor (FGF) have been identified as activators of angiogenesis $[13,14]$.

VEGF is one of the most effective angiogenic components that play a critical role in neovascularization. The VEGF family consists of six famous members. VEGF-A is a glycoprotein that has several molecular isoforms and is also called VEGF or VPE (vascular permeability factor). VEGF-stimulated signal activates various downstream proteins such as phospholipase $\mathrm{C}$, PI3-K, GAP, the Ras GTPase-activating protein, p38MAPK, ERK, p125FAK, and inducing cell progression. VEGF-A is a strong and particular mitogen for vascular endothelial cells that stimulates the angiogenesis pathways and is up-regulated in different tumors $[15,16]$.

FGF is another essential molecule that regulates angiogenesis. The FGF family includes nine sperate members. FGF-1 (acidic, aFGF) and FGF-2 (basic, bFGF) are indicated as potent activators of angiogenesis. FGF binds to specific receptors resulting in activation of downstream signal transduction cascade and play important roles in wound healing and embryonic development. Moreover, some FGF signaling promotes tumor angiogenesis and growth [17]. Additionally, VEGF and bFGF act as antiapoptotic agents for the neovascularization, because they stimulate antiapoptotic molecules (like Bcl2) expression and increase endothelial cell survival $[18,19]$.

In the current study, we evaluated the expression of DDR1 that induced by collagen type I and inhibited by siRNA in non-small cell lung cancer cells. Then, we investigated the expression of VEGF-A, FGF-1, and FGF-2 to find whether expression alteration in angiogenic factors were correlated with expression of DDR1 in NSCLC.

\section{Material And Methods}

\section{Cell lines and culture conditions}


We purchased the human NSCLC adenocarcinoma cell lines (A549 and Calu-3) from the National Cell Bank of Iran (Pasteur Institute of Iran, Tehran) and maintained them according to the manufacturer's instructions. Two cell lines were cultured and grown in Dulbecco's modified eagle medium (DMEM) with a high concentration of glucose supplemented with 10\% fetal bovine serum (FBS) (Gibco, USA), $100 \mathrm{U} / \mathrm{ml}$ penicillin, and $100 \mu \mathrm{g} / \mathrm{ml}$ streptomycin at $37^{\circ} \mathrm{C}$ in humidified air with $5 \% \mathrm{CO} 2$.

\section{Preparation of collagen I coated plate and collagen I stimulation}

For Coating cell culture dishes with rat tail collagen I (Roche, Mannheim, Germany), the lyophilized Collagen was dissolved in sterile $0.2 \%$ acetic acid $(\mathrm{v} / \mathrm{v})$ according to the manufacturer's instructions. Then, sufficient diluted collagen was added to coat dishes $\left(5-10 \mu \mathrm{g} / \mathrm{cm}^{2}\right)$ surface and Carefully spread the collagen solution on the bottom of the culture dishes. After that, the plates were incubated for about 60 min at +15 to $+25^{\circ} \mathrm{C}$ in the laminar flow hood. Finally, the coated surface was washed with medium or buffer. For DDR1 activation by collagen I, cells were implanted in the plates and grown on collagen I or control at various time points.

\section{Knockdown transfection}

cells were split to $60-70 \%$ confluence in each well of 6-well plates. The next day, transfection of the siDDR1 (ON-TARGETplus siRNA, Dharmacon, Colorado, USA) and control small interfering RNA (ONTARGETplus Non-targeting Control siRNA, Dharmacon, Colorado, USA) into A549 and Calu-3 cells were performed using DharmaFECT Transfection Reagent (Dharmacon, Colorado, USA) according to manufacturer protocol with $25 \mathrm{nM}$ final concentration of siRNAs. Cells were grown in serum-free medium for six hours. Then, we added $10 \%$ FBS to the complete medium, and cells were grown with $10 \%$ FBS for $42 \mathrm{~h}$.

\section{RNA extraction}

Total RNA was isolated from each cell line after 2, 4, 8, 12, and 16 hours of collagen induction and $48 \mathrm{~h}$ siRNA transfection by TRIzol reagent (Invitrogen, Carlsbad, CA, USA) according to the manufacturer's instructions. The concentration of RNA and A260/280 ratio were evaluated using a NanoDrop Spectrophotometer (Thermo Fisher Scientific, Waltham, USA). RNA quality was determined via agarose gel electrophoresis.

\section{Quantitative real-time PCR}

For analysis of DDR1, VEGF-A, FGF-1, FGF-2, and GAPDH mRNA expression, $1 \mu \mathrm{g}$ total RNA was reverse transcribed using random primers and RevertUP ${ }^{\text {TM }}$ II Reverse Transcriptase (Biotechrabbit ${ }^{\text {TM }}$ cDNA Synthesis Kit, Hennigsdorf, Germany) according to the manufacturer's protocol. Quantitative Real-time polymerase chain reaction (qRT-PCR) was performed using the SYBR Green assay in the Corbett RotorGene 6000 Real-time PCR Machine (Qiagen, Hilden, Germany). The comparative threshold cycle method $\left(\Delta \Delta \mathrm{C}_{\mathrm{t}}\right)$ was used to quantify relative amounts of transcripts with GAPDH as an internal control. Reactions were performed in duplicate, and the results of three independent experiments were subjected to 
statistical analysis. The authenticity of the amplified products was evaluated by agarose gel electrophoresis.

\section{Western blot}

Cells were implanted on $10 \mathrm{~cm}$ plates coated with $1 \mathrm{mg} / \mathrm{mL}$ collagen and fibronectin (control). Then, after acceptable confluency, they were lysed with Pierce RIPA buffer (Thermo Fisher Scientific, Waltham, USA) containing $10 \mu \mathrm{g} / \mathrm{mL}$ Na2VO4, $10 \mu \mathrm{g} / \mathrm{mL}$ phenyl methyl sulfonyl fluoride (PMSF), and $20 \mu \mathrm{g} / \mathrm{mL}$ protease inhibitor cocktail ( $\mu \mathrm{g} / \mathrm{ml}$ leupeptin and $10 \mu \mathrm{g} / \mathrm{ml}$ aprotinin). Protein yields were assigned by a BCA Protein assay kit (Thermo Fisher Scientific, Waltham, USA), and $40 \mu \mathrm{g}$ of protein was loaded with 1X SDS sample buffer onto $10 \%$ or $8 \%$ polyacrylamide gels. Gels were transferred onto $0.45 \mu \mathrm{m}$ nitrocellulose membranes (Bio-Rad, California, USA) and blocked in 5\% BSA. Next, Membranes were incubated overnight with primary antibodies at $4^{\circ} \mathrm{C}$ in $2.5 \%$ BSA in TBS with $0.1 \%$ Tween-20. Membranes were incubated with respective secondary antibody for $1 \mathrm{~h}$ in TBS with $0.1 \%$ Tween-20 at room temperature. Finally, Image Studio (Mandel) was used for the detection and analysis of immunoblots. Goat anti-DDR1 antibodies were purchased from R\&D Systems (Minneapolis, USA). $\beta$-actin (MABT825) primary mouse antibodies were obtained from Millipore Sigma (Burlington, Massachusetts, USA).

\section{Angiogenic factors analysis}

To evaluate the angiogenic factor concentrations in collagen type I stimulated cells, the cell lysate was collected from the collagen I coated plates and control plates after $16 \mathrm{~h}$. The cell lysate was frozen at $-80^{\circ} \mathrm{C}$ and shipped on dry ice to Eve Technologies (Calgary, AB, Canada) for analysis using Human Angiogenesis 17-Plex Discovery Assay.

\section{Statistical analysis}

The results were analyzed using the GraphPad Prism 8.0 statistical software (GraphPad Software Inc., San Diego, USA). The expression levels of mRNA in each cell sample were normalized to those of GAPDH. The significance of differences between groups was assessed using the unpaired Student's t-test or analysis of variance (ANOVA). P values $<0.05$ ( $^{*}$ ) were considered significant. Our Data were illustrated as mean \pm standard deviation (SD) with at least three independent experiments.

\section{Results}

Upregulation and downregulation of DDR1 by collagen I stimulation and siRNA transfection in NSCLC cell lines

To determine whether collagen I could promote the DDR1 expression in NSCLC cells, we stimulated A549 and Calu-3 cells with collagen I. A549 and Calu-3 cells were incubated in collagen I-coated plates and control plates for various times, and then total RNA was extracted to perform Real-time quantitative PCR. The results showed that mRNA expression of DDR1 was significantly increased in A549 and Calu-3 cells treated with collagen I compared to control (Fig. 1a, C). Also, the total protein expression of DDR1 was detected using Western blot analysis (Fig. 1d). Our data revealed that mRNA and protein expressions of 
DDR1 in A549 and Calu-3 cells were induced in a time-dependent mode, and after $16 \mathrm{~h}$ of collagen I stimulation reached the highest expression. The cells were transfected with four different DDR1 siRNAs to reduce the expression of DDR1. The DDR1 mRNA expression levels were significantly decreased in A549 and Calu-3 cells after being treated with siDDR1 compared to Non-targeting control siRNA transfected cells (Fig. 1b, c).

\section{Effects of DDR1 on the angiogenic factors expression in NSCLC cell lines}

To investigate the relationship between expression of DDR1 and angiogenic factors in two NSCLC cell lines (A549 and Calu-3 cells), we have transiently overexpressed DDR1 with collagen I stimulation at various time points. Also, knockdown of DDR1 was performed in A549 and Calu-3 cell lines using DDR1 siRNA sequences and non-targeting control. The Real-time quantitative PCR results showed that the mRNA expression levels of DDR1 in A549 and Calu-3 cells were significantly increased after 16 hours compared with unstimulated cells. Furthermore, DDR1 mRNA expressions in A549 and Calu-3 were significantly decreased after siRNA transfection. Therefore, to confirm the effects of DDR1 on VEGF-A, FGF-1, and FGF-2 expression, we evaluated the expression levels of VEGF-A, FGF-1, and FGF-2 in the A549 and Calu-3 cells after stimulation of collagen I and siRNA transfection. In the following assays, VEGF-A (Fig. 2a, c) and FGF-2 (Fig. 3a, c) mRNA expression levels were significantly increased after DDR1 overexpression in these two cell lines in a time-dependent mode, and after $16 \mathrm{~h}$ of collagen I stimulation reached the highest expression. Moreover, in both the cell lines tested, knockdown of DDR1 affected the mRNA levels of VEGF-A and FGF-2, significantly decreased VEGF-A (Fig. 2b, c) and FGF-2 (Fig. 3b, c) expressions. In contrast, the observed difference in expression levels of FGF-1 was not significant statistically in both cell lines (Fig. 4a, b, c). Our results demonstrate that inhibition of DDR1 downregulates VEGF-A and FGF-2, whereas stimulation of DDR1 up-regulates them.

\section{angiogenic factors Analysis}

we used Human Angiogenesis 17-Plex Discovery Assay to analyze the production of angiogenic factors (VEGF-A, FGF-1, and FGF-2) in A549 and Calu-3 cells stimulated with collagen type I. According to the fluorescent intensity results in two cell lines, the concentrations of VEGF-A and FGF-2 increased significantly after collagen I stimulation but FGF-1 (Fig. 5a, b, c) did not increase.

\section{Conclusions}

In the group of human cancers, lung cancer is the most common in both sexes, with a high incidence, and has one of the worst prognoses between cancers. Most lung cancer cases are NSCLC and have the lowest 5-year survival rate of leading human cancers. So, new and effective targeted therapies are essential $[1,20]$. 
DDR1 is a tyrosine kinase receptor with the unique ability among RTKs to respond to several collagen types $[6,7]$. The DDR1 has been shown to regulate various cellular signaling pathways, cell proliferation, adhesion, migration, matrix remodeling. Thus, dysregulation of DDR1 may lead to metastatic cancer progressions. Gather evidence confirmed overexpression of DDR1 in invasive tumors that showed enhanced migration and invasion in cancer cells [21-24]. Upregulation of DDR1 in NSCLC tissues indicated that increased DDR1 expression might be involved in the development and progression of NSCLC. The DDR1 has been reported to be basically activated by collagens I-IV and VIII. Accordingly, collagen type I increases DDR1 expression in primary human lung fibroblasts at specific doses and times. In addition, collagen type I activates the DDR1 pathway to promote EMT by activation of MMP-9 and contribute to NSCLC cell invasion [21, 25]. DDR1 stimulates MAPK signaling by activating ERK1/2 or the JNK pathway in various cell lines. In addition, DDR1 activation has been shown to induce PI3 kinase/Akt and NFKB signaling pathways in cancer cells [9].

Tumor growth and metastasis depend on angiogenesis triggered by chemical signals from tumor cells in a phase of rapid growth. At the onset of angiogenesis, proangiogenic factors, including VEGF and related molecules, increase. Growth factors and cytokines that control angiogenesis are included VEGF, FGF, angiopoeintins, TNF- $\alpha$ and TNF- $\beta$. VEGF acts as a major key to angiogenesis in the early stages of both physiological and pathological conditions. Up-regulation of FGF-2 (basic FGF) has been shown to increase angiogenic activity and also cooperate with other angiogenic molecules including VEGF. Because VEGF and FGF have been shown to be abundantly produced in a variety of angiogenic diseases, they can be screened for appropriate therapeutic purposes [26].

Accordingly, various studies have been performed on treatment methods to inhibit angiogenesis and thus suppress tumor growth. For example, treatment with relevant anti-miRNA suppressors can neutralize the expression of miRNA antiangiogenic target genes by increasing miRNA expression and inhibit the angiogenic response. Overexpression of VEGF or bFGF increases miR-132 expression, which induces Ras activity and cell proliferation by inhibiting p120RasGAP (the Ras GTPase activating protein) expression. Thus, anti-miR-132 expression retains the expression of p120RasGAP that inhibits Ras activity and suppresses angiogenesis cascades of several growth factors [27].

Another study has shown that DDR1 expression stimulates the formation of tubular structures in HUVEC cells culture medium with increased DDR1 expression [28]. However, the mechanism of DDR1 angiogenesis stimulation is not yet known and its determination requires further studies. Also, the study of DDR1 and its effect on angiogenesis in lung cancer can introduce it as a suitable biomarker and therapeutic target.

In the present study, we showed that collagen I could significantly promote DDR1 expression in lung adenocarcinoma cells (A549 and Calu-3). Also, the expression of VEGF-A and FGF-2 were significantly increased by DDR1 up-regulation. Therefore, DDR1 expression is a crucial clinically molecular target for NSCLC patients. In conclusion, our findings demonstrate that DDR-1 proteins play a major role in promoting angiogenesis by regulating the expression of angiogenic factors: VEGF-A and bFGF in non- 
small cell lung cancer cells. The DDR1-dependent angiogenesis provides a new paradigm in lung tumorigenesis which could have an impact on its treatment.

\section{Declarations}

\section{Conflict of interest}

The authors declare that they have no conflict of interest

\section{Funding}

This research was funded by the National Institute of Genetic Engineering and Biotechnology (Grant number 787)

Author Massoud Houshmand has received research support from the National Institute of Genetic Engineering

and Biotechnology.

\section{Competing Interests}

The authors have no relevant financial or non-financial interests to disclose.

\section{Author Contributions}

Zahra Damavandi: study concept and experimental design, acquisition of data; analysis and interpretation of data; drafting of the manuscript; statistical analysis.

Pardis Riahi: assisted in the experiments, experimental trouble shooting and assisted in interpretation of data.

Tayebeh Majidizadeh: material preparation, technical and experimental support.

Massoud Houshmand: study concept and design; study supervision; study support; critical revision of the manuscript.

The first draft of the manuscript was written by zahra damavandi and all authors commented on previous versions of the manuscript. All authors read and approved the final manuscript.

\section{References}

1. Siegel RL, Miller KD, Jemal A. (2019) Cancer statistics 2019. CA: A Cancer Journal for Clinicians 69:734. https://doi.org/10.3322/caac.21551.

2. Molina JR, et al. (2008) Non-Small Cell Lung Cancer: Epidemiology, Risk Factors, Treatment, and Survivorship. Mayo Clinic Proceedings 83:584-594. https://doi.org/10.4065/83.5.584. 
3. Yang IA, Holloway JW, Fong KM. Genetic susceptibility to lung cancer and co-morbidities. J Thorac Disease. 2013;5:454-62. https://doi.org/10.3978/j.issn.2072-1439.2013.08.06.

4. Jing $\mathrm{H}$, Song $\mathrm{J}$, Zheng J. Discoidin domain receptor 1 : New star in cancer-targeted therapy and its complex role in breast carcinoma. Oncol Lett. 2018;15:3403-8. https://doi.org/10.3892/ol.2018.7795.

5. Valiathan RR, et al. Discoidin Domain Receptor Tyrosine Kinases: new players in cancer progression. Cancer Metastasis Rev. 2012;31:295-321. https://doi.org/10.1007/s10555-012-9346-z.

6. Vogel W, et al. The Discoidin Domain Receptor Tyrosine Kinases Are Activated by Collagen. Mol Cell. 1997;1:13-23. https://doi.org/10.1016/S1097-2765(00)80003-9.

7. Carafoli F, Hohenester E. Collagen recognition and transmembrane signalling by discoidin domain receptors. Biochimica et Biophysica Acta (BBA) -. Proteins and Proteomics. 2013;1834:2187-94. https://doi.org/10.1016/j.bbapap.2012.10.014.

8. Borza CM, Pozzi A. Discoidin domain receptors in disease. Matrix Biol. 2014;34:185-92. https://doi.org/10.1016/j.matbio.2013.12.002.

9. Leitinger B. Discoidin domain receptor functions in physiological and pathological conditions. Int Rev cell Mol biology. 2014;310:39-87. https://doi.org/10.1016/B978-0-12-800180-6.00002-5.

10. Prakoura N, Chatziantoniou C. Periostin and Discoidin Domain Receptor 1: New Biomarkers or Targets for Therapy of Renal Disease. Front Med. 2017;4:52. https://doi.org/10.3389/fmed.2017.00052.

11. Rammal H, et al. Discoidin Domain Receptors: Potential Actors and Targets in Cancer. Front Pharmacol. 2016;7:55. https://doi.org/10.3389/fphar.2016.00055.

12. Shimada $\mathrm{K}$, et al. Prostate cancer antigen-1 contributes to cell survival and invasion though discoidin receptor 1 in human prostate cancer. Cancer Sci. 2008;99:39-45. https://doi.org/10.1111/j.13497006.2007.00655.x.

13. Folkman J. Tumor Angiogenesis: Therapeutic Implications. N Engl J Med. 1971;285:1182-6. https://doi.org/10.1056/nejm197111182852108.

14. Nishida N, et al. Angiogenesis in cancer. Vasc Health Risk Manag. 2006;2:213-9. https://doi.org/10.2147/vhrm.2006.2.3.213.

15. Ferrara N, Gerber HP. (1999) Vascular Endothelial Growth Factor Molecular and Biological Aspects. in Advances in Organ Biology, E.E. Bittar, Editor. Elsevier. pp 25-57.

16. Dvorak HF. Vascular permeability factor/vascular endothelial growth factor: a critical cytokine in tumor angiogenesis and a potential target for diagnosis and therapy. J Clin Oncol. 2002;20:4368-80. https://doi.org/10.1200/jco.2002.10.088.

17. Tonini T, Rossi F, Claudio PP. Molecular basis of angiogenesis and cancer. Oncogene. 2003;22:654956. https://doi.org/10.1038/sj.onc.1206816.

18. Gerber HP, Dixit V, Ferrara N. Vascular endothelial growth factor induces expression of the antiapoptotic proteins Bcl-2 and A1 in vascular endothelial cells. J Biol Chem. 1998;273:13313-6. 
https://doi.org/10.1074/jbc.273.21.13313.

19. Araki $S$, et al. Role of protein kinase $C$ in the inhibition by fibroblast growth factor of apoptosis in serum-depleted endothelial cells. Biochem Biophys Res Commun. 1990;172:1081-5. https://doi.org/10.1016/0006-291x(90)91557-9.

20. Yang $\mathrm{SH}$, et al. Discoidin domain receptor 1 is associated with poor prognosis of non-small cell lung carcinomas. Oncol Rep. 2010;24:311-9. https://doi.org/10.3892/or_00000861.

21. Miao L, et al. Discoidin domain receptor 1 is associated with poor prognosis of non-small cell lung cancer and promotes cell invasion via epithelial-to-mesenchymal transition. Med Oncol. 2013;30:626. https://doi.org/10.1007/s12032-013-0626-4.

22. L'Hôte C, Thomas GPH, Ganesan TS. Functional analysis of discoidin domain receptor 1: effect of adhesion on DDR1 phosphorylation. Faseb j. 2002;16:234-6. https://doi.org/10.1096/fj.01-0414fje.

23. Matada GSP, et al. DDR1 and DDR2: a review on signaling pathway and small molecule inhibitors as an anticancer agent. Med Chem Res. 2021;30:535-51. https://doi.org/10.1007/s00044-020-02694-2.

24. Ford $\mathrm{CE}$, et al. Expression and mutation analysis of the discoidin domain receptors 1 and 2 in nonsmall cell lung carcinoma. Br J Cancer. 2007;96:808-14. https://doi.org/10.1038/sj.bjc.6603614.

25. Ruiz PA, Jarai G. Collagen I induces discoidin domain receptor (DDR) 1 expression through DDR2 and a JAK2-ERK1/2-mediated mechanism in primary human lung fibroblasts. J Biol Chem. 2011;286:12912-23. https://doi.org/10.1074/jbc.M110.143693.

26. Furuya M, et al. Pathophysiology of Tumor Neovascularization. Vasc Health Risk Manag. 2005;1:277-90. https://doi.org/10.2147/vhrm.2005.1.4.277.

27. Anand $S$, et al. MicroRNA-132-mediated loss of p120RasGAP activates the endothelium to facilitate pathological angiogenesis. Nat Med. 2010;16:909-14. https://doi.org/10.1038/nm.2186.

28. Song J, et al. Discoidin domain receptor 1 (DDR1), a promising biomarker, induces epithelial to mesenchymal transition in renal cancer cells. Tumour Biol. 2016;37:11509-21. https://doi.org/10.1007/s13277-016-5021-2.

\section{Figures}


A

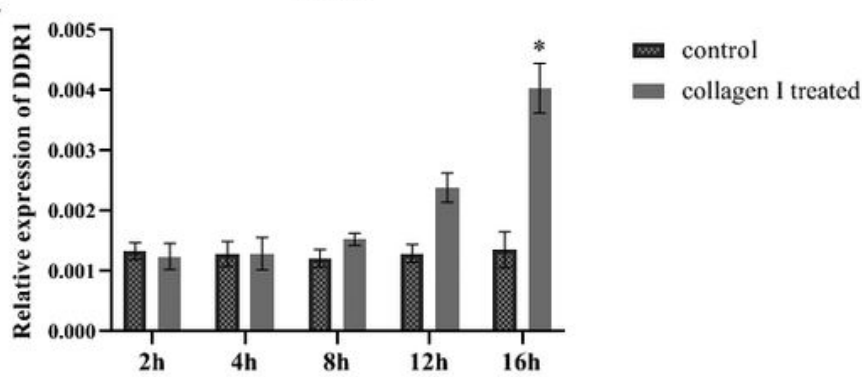

Calu-3

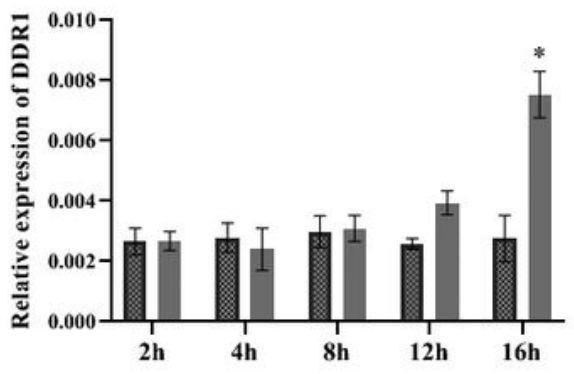

B

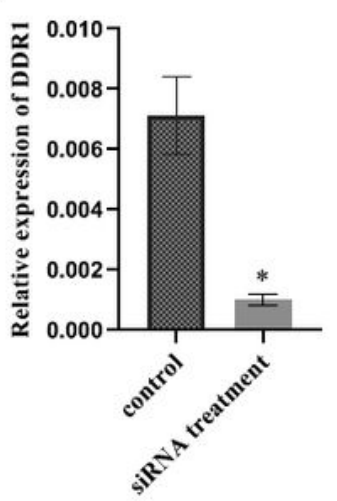

A549

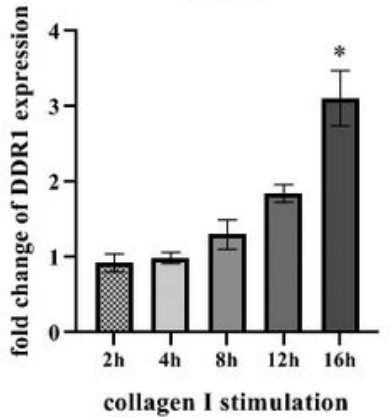

Calu-3

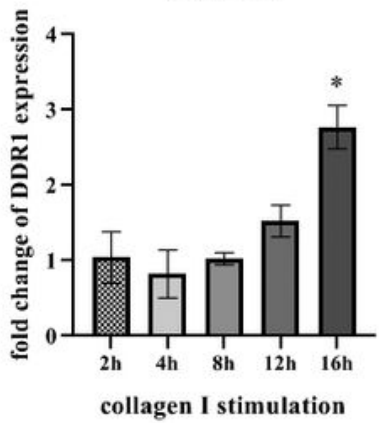

Calu-3

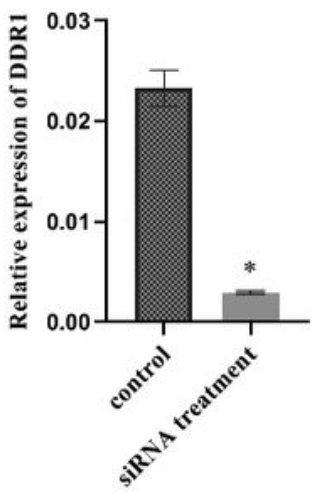

D

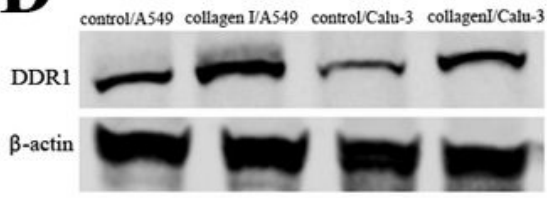

Figure 1

Expression of DDR1 by collagen I simulation and after siRNA transfection in A549 and Calu-3 cells. a. Expression analysis of DDR1 mRNA in A549 and Calu-3 cells were incubated with collagen I at various times by real-time PCR. DDR1 mRNA expressions were increased significantly after 16 hours under the collagen I stimulation. Also, the expression fold changes were calculated relative to unstimulated control A549 and Calu-3 cells after normalizing with GAPDH. b. Analysis of DDR1 expressions after siRNA 
transfection in A549 and Calu-3 cells using real-time PCR. DDR1 mRNA expressions were decreased significantly after si-DDR1 transfection compared with non-targeting Control siRNA. c. the size and uniqueness of PCR products following agarose gel electrophoresis. $\mathbf{d}$. western blot analysis of DDR1 protein expressions in A549 and Calu-3 cells were incubated with collagen I and fibronectin as control after $16 \mathrm{~h}$ that DDR1 expressions were enhanced under the collagen I stimulation. All experiments were conducted in three biological and two technical replicates. ${ }^{\star} P<0.05$.

A
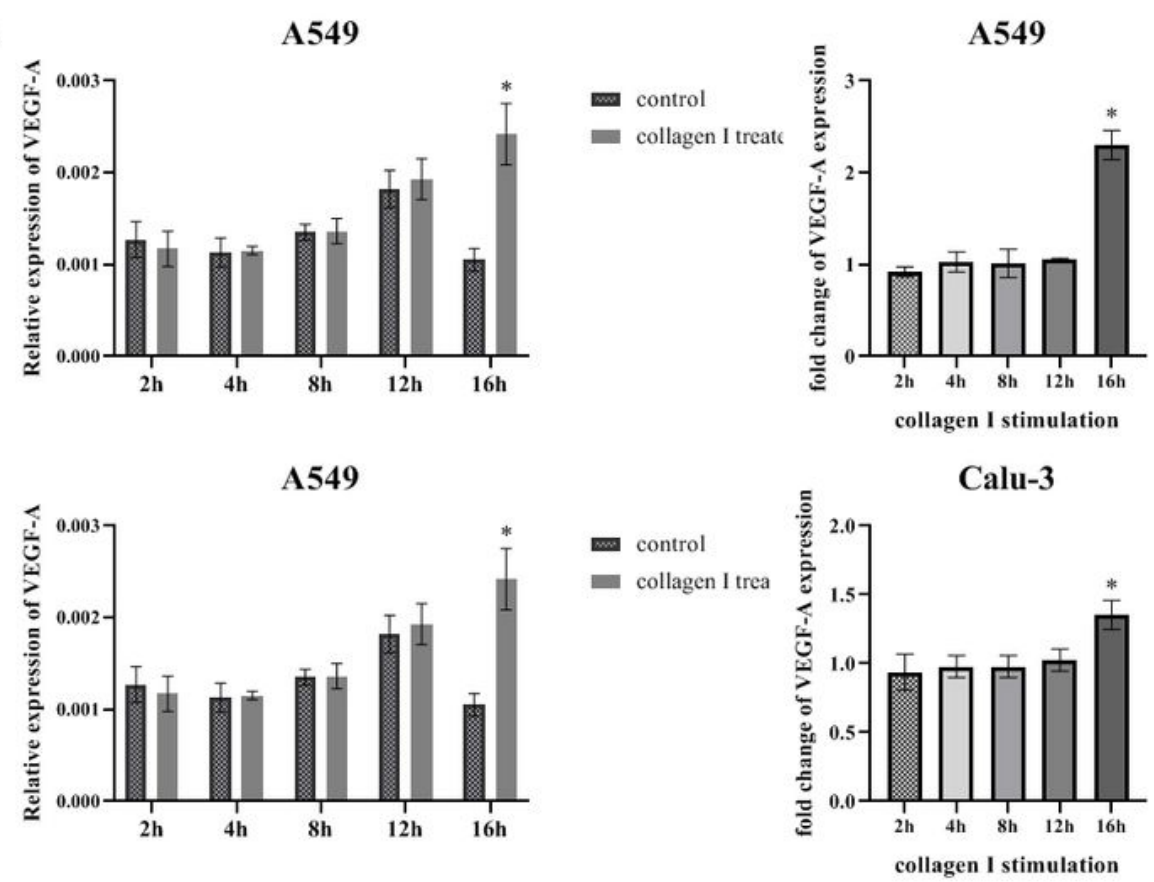

B
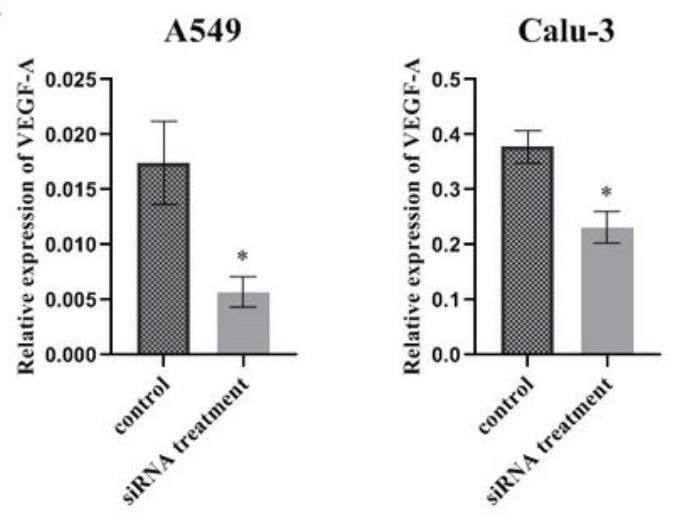

C

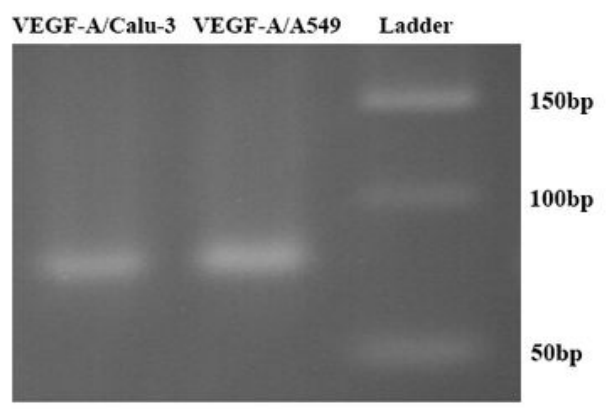




\section{Figure 2}

DDR1 promotes the expression of VEGF-A in A549 and Calu-3 cells. Real-time PCR was conducted to evaluate the VEGF-A expression in NSCLC cells stimulated by collagen I in a time-dependent manner and after DDR1 siRNA transfection. a. Histograms show relative VEGF-A mRNA expressions were significantly increased in A549 and Calu-3 cells after 16 hours of collagen I stimulation. Also, the expression fold changes were calculated relative to unstimulated control A549 and Calu-3 cells after normalizing with GAPDH. b. Histograms show relative VEGF-A mRNA expressions were significantly decreased after siDDR1 transfection versus non-targeting control siRNA in A549 and Calu-3 cells. c. The size and uniqueness of PCR products following agarose gel electrophoresis. All experiments were conducted in three biological and two technical replicates. ${ }^{*} P<0.05$. 
A

A549

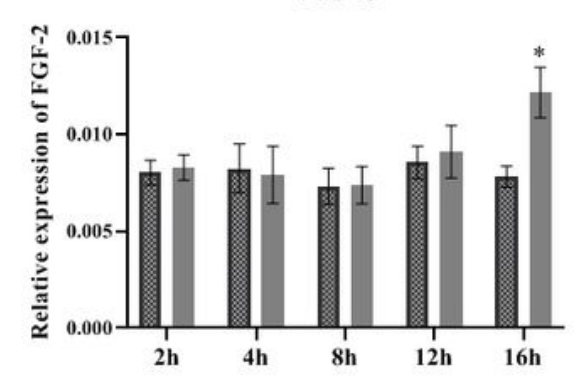

Calu-3

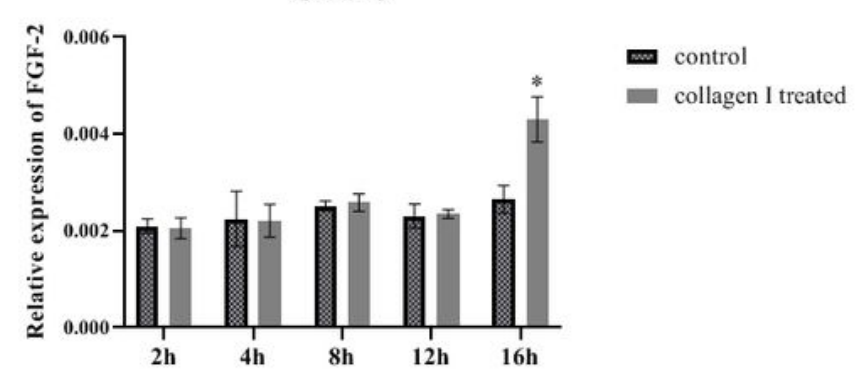

A549

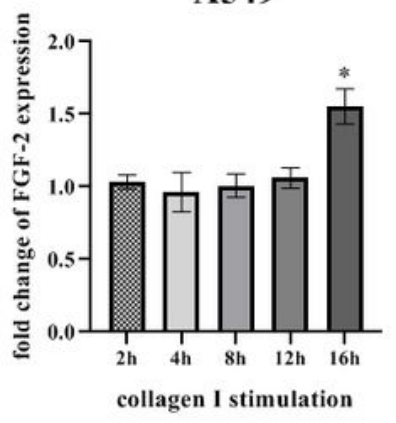

Calu-3

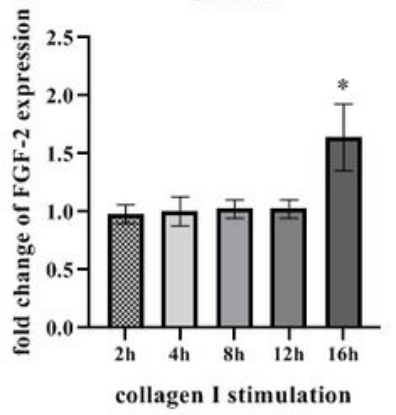

B

A549

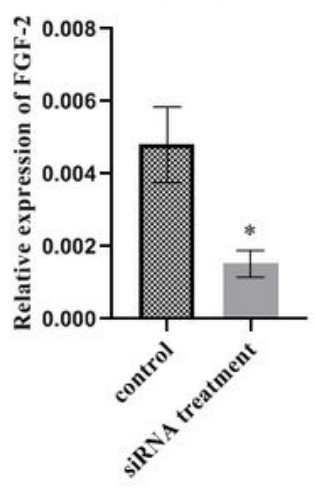

C
Calu-3

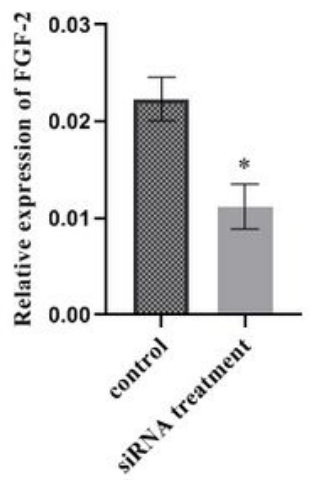

200bp

150bp

100bp

50bp

\section{Figure 3}

DDR1 promotes the expression of FGF-2 in A549 and Calu-3 cells. Real-time PCR was conducted to evaluate the FGF-2 expression in NSCLC cells stimulated by collagen I in a time-dependent manner and after DDR1 siRNA transfection. a. Histograms show relative FGF-2 mRNA expressions were significantly increased in A549 and Calu-3 cells after 16 hours of collagen I stimulation. Also, the expression fold changes were calculated relative to unstimulated control A549 and Calu-3 cells after normalizing with 
GAPDH. b. Histograms show relative FGF-2 mRNA expressions were significantly decreased after si-DDR1 transfection versus non-targeting control siRNA in A549 and Calu-3 cells. c. The size and uniqueness of PCR products following agarose gel electrophoresis. All experiments were conducted in three biological and two technical replicates. ${ }^{*} P<0.05$.

A

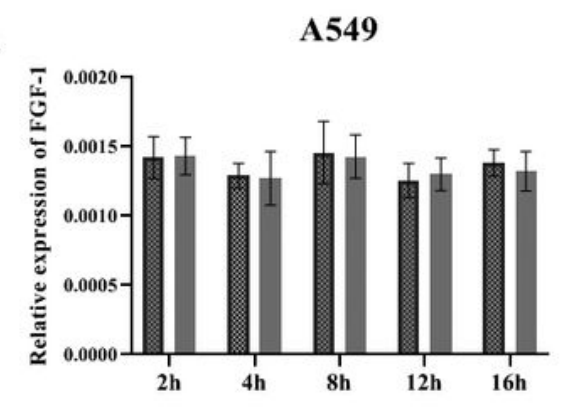

Calu-3

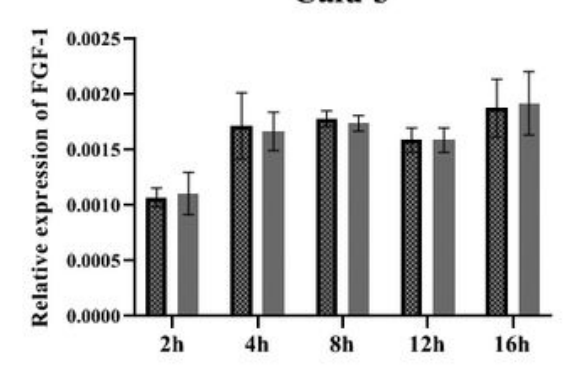

B

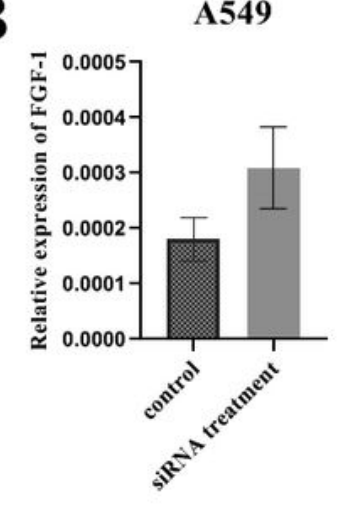

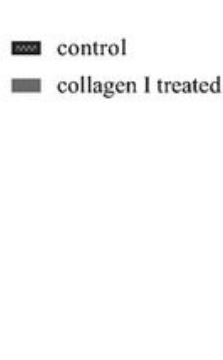

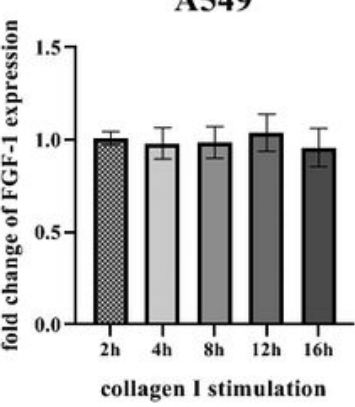

Calu-3

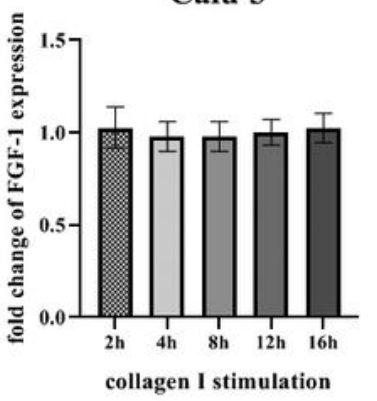

C

FGF-1/Calu-3 FGF-1/A549 Ladder

Figure 4 
Evaluation of FGF-1 expression in A549 and Calu-3 cells. Real-time PCR was conducted to evaluate the FGF-1 expression in NSCLC cells stimulated by collagen I in a time-dependent manner and after DDR1 siRNA transfection. a. Histograms show relative FGF-1 mRNA expressions were not significantly increased in A549 and Calu-3 cells after 16 hours of collagen I stimulation. b. Histograms show relative FGF-1 mRNA expressions were not significantly decreased after si-DDR1 transfection versus nontargeting control siRNA in A549 and Calu-3 cells. c. The size and uniqueness of PCR products following agarose gel electrophoresis. All experiments were conducted in three biological and two technical replicates. ${ }^{\star} P<0.05$. 
A

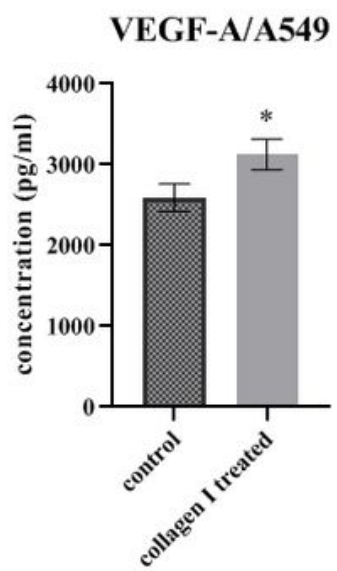

VEGF-A/Calu-3

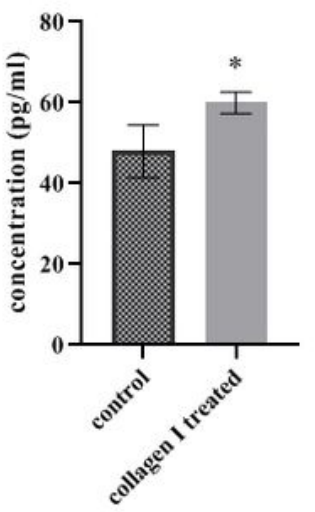

B

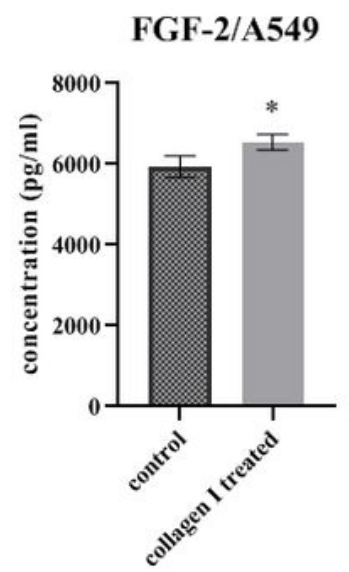

FGF-2/Calu-3

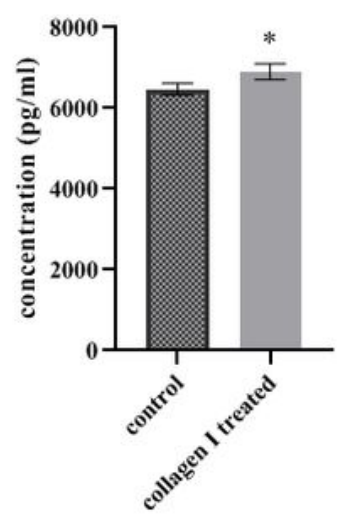

C
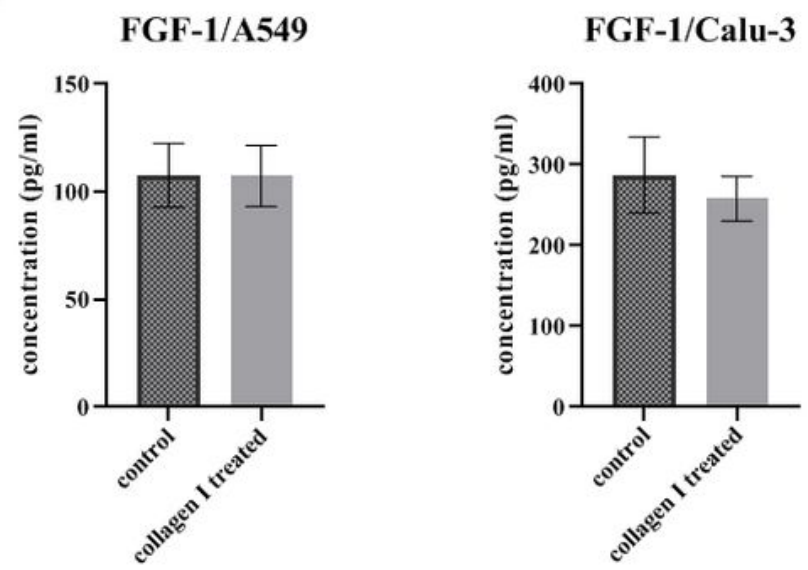

Figure 5

angiogenic factors analysis. Evaluation of angiogenic factors concentration $(\mathrm{pg} / \mathrm{mL})$ in A549 and Calu-3 cell lysates after $16 \mathrm{~h}$ collagen I stimulation. Histograms show fluorescent intensity of VEGF-A (a), FGF-2 (b) and FGF-1 (c) in both cell lines. We observed a significant increase in VEGF-A and FGF-2 concentrations. ${ }^{*} P<0.05$. 
Page 18/18 